\title{
Mutations in the Drosophila dTAK1 gene reveal a conserved function for MAPKKKs in the control of rel/NF-kB-dependent innate immune responses
}

\author{
Sheila Vidal, ${ }^{1,3}$ Ranjiv S. Khush, ${ }^{1,3}$ François Leulier, ${ }^{1,3}$ Phoebe Tzou, ${ }^{1}$ Makoto Nakamura, ${ }^{2}$ \\ and Bruno Lemaitre ${ }^{1,4}$ \\ ${ }^{1}$ Centre de Génétique Moléculaire, CNRS, 91198 Gif-sur-Yvette, France; ${ }^{2}$ National Institute for Basic Biology, \\ Okasaki 444-8585, Japan
}

In mammals, TAK1, a MAPKKK kinase, is implicated in multiple signaling processes, including the regulation of NF-KB activity via the IL1-R/TLR pathways. TAK1 function has largely been studied in cultured cells, and its in vivo function is not fully understood. We have isolated null mutations in the Drosophila dTAK1 gene that encodes dTAK1, a homolog of TAK1. dTAK1 mutant flies are viable and fertile, but they do not produce antibacterial peptides and are highly susceptible to Gram-negative bacterial infection. This phenotype is similar to the phenotypes generated by mutations in components of the Drosophila Imd pathway. Our genetic studies also indicate that dTAK1 functions downstream of the Imd protein and upstream of the IKK complex in the Imd pathway that controls the Rel/NF-kB like transactivator Relish. In addition, our epistatic analysis places the caspase, Dredd, downstream of the IKK complex, which supports the idea that Relish is processed and activated by a caspase activity. Our genetic demonstration of dTAK1's role in the regulation of Drosophila antimicrobial peptide gene expression suggests an evolutionary conserved role for TAK1 in the activation of Rel/NF-кB-mediated host defense reactions.

[Key Words: Innate immunity; antimicrobial peptide; rel/NF-кB; Toll; TAK1; caspase]

Received March 20, 2001; revised version accepted June 7, 2001.

Insects rely on multiple strategies to defend themselves against microbial infection: (a) they activate proteolytic signaling cascades that lead to melanization and coagulation reactions at injury sites and around foreign objects; (b) they utilize blood cells to either phagocytose or encapsulate invading microbes; and (c) they produce numerous antimicrobial peptides (for review, see Hoffmann and Reichhart 1997; Khush and Lemaitre 2000). Antimicrobial peptides are synthesized in the fat body (an ana$\log$ of the mammalian liver) in response to systemic infections and in several epithelial tissues in response to local infections (Ferrandon et al. 1998; Tzou et al. 2000; Onfelt Tingvall et al. 2001). Seven distinct classes of peptides have been identified in Drosophila: Drosomycin, Metchnikowin, Diptericin, Drosocin, Cecropin, Defensin, and Attacin. Drosomycin is mainly active against

\footnotetext{
${ }^{3}$ These authors contributed equally to this work.

${ }^{4}$ Corresponding author.

E-MAIL lemaitre@cgm.cnrs-gif.fr; FAX 33-1-69-82-43- 86.

Article and publication are at http://www.genesdev.org/cgi/doi/10.1101/ $\operatorname{gad} .203301$.
}

fungi; Metchnikowin shows both antifungal and antibacterial activities; the other peptides are mainly active against bacteria (for review, see Hoffmann and Reichhart 1997). Rel/Nuclear Factor (NF)-кB-like transcription factors are the major regulators of antimicrobial peptide gene expression in Drosophila, and their activity is modulated by signaling pathways that are similar to the interleukin 1-receptor (IL1-R) in mammals (for review, see Engstrom 1999; Anderson 2000; Khush et al. 2001). These similarities indicate an evolutionary link between the control of antimicrobial peptide gene expression and the control of mammalian innate immune responses (Hoffmann et al. 1999; Aderem and Ulevitch 2000).

The Toll signaling pathway, which was first identified as a regulator of embryonic dorsal-ventral patterning, is one regulator of antimicrobial peptide gene expression (Lemaitre et al. 1996). Upon infection, the Spaetzle (Spz) protein is cleaved to generate a ligand for the Toll transmembrane receptor protein; Toll binding by Spz stimulates the degradation of the Inhibitory (I)-кB homolog, Cactus, and the nuclear translocation of the Rel proteins Dorsal and Dorsal-like immunity factor (Dif). Dorsal and 
Dif appear to function redundantly to regulate antimicrobial peptide gene expression in larvae, but in adults, Dif is required for Toll-mediated antimicrobial peptide gene induction (Manfruelli et al. 1999; Meng et al. 1999; Rutschmann et al. 2000a). Loss-of-function mutations in genes of the Toll signaling cascade $(s p z$, Toll, pelle, and tube) impair the induction of the antifungal peptide gene Drosomycin, and in flies carrying a gain-of-function Toll mutation that activates the Toll pathway, Drosomycin is constitutively expressed (Lemaitre et al. 1996). Tolldeficient flies resist bacterial infections but are highly susceptible to fungal infections, demonstrating that the immune responses regulated by the Toll pathway are required to resist fungal infection (Lemaitre et al. 1996).

The second pathway regulating antimicrobial peptide gene expression in flies was initially identified by a mutation in the immune deficiency (imd) gene that results in susceptibility to Gram-negative bacterial infection and an impairment of antibacterial peptide gene expression (Lemaitre et al. 1995). imd encodes a homolog of the mammalian Receptor Interacting Protein (RIP) (P. Georgel et al., in prep.), and molecular studies have isolated four additional factors that appear to define the Imd pathway: Relish, a third Drosophila Rel protein (Hedengren et al. 1999); two members of a Drosophila IкB kinase (IKK) complex, that is, the kinase DmIKK $\beta$ and a structural component DmIKK $\gamma$ (Kim et al. 2000; Rutschmann et al. 2000b; Silverman et al. 2000; Lu et al. 2001) and Dredd, a caspase (Elrod-Erickson et al. 2000; Leulier et al. 2000). Relish is a homolog of the mammalian P100 and P105 compound Rel proteins that contain both Rel domains and inhibitory ankyrin domains (Hedengren et al. 1999). A receptor for the Imd pathway has not been identified, but infection triggers Relish cleavage and nuclear translocation of the Rel domain (Stöven et al. 2000). Relish cleavage requires DmIKK $\beta$ activity, indicating that, like the mammalian IKK complex that functions in the IL1-R and TNF-R pathways, the Drosophila IKK complex regulates Rel protein activity (Silverman et al. 2000). In contrast to P100 and P105 processing, however, Relish cleavage is not blocked by proteasome inhibitors but does require a functional Dredd gene, suggesting that Dredd may cleave Relish directly after infection (Stöven et al. 2000). Like imd, mutations in DmIKK $\beta, D m I K K \gamma, D r e d d$, and Relish affect antibacterial peptide gene expression after infection and induce susceptibility to Gram-negative bacterial infections (Hedengren et al. 1999; Leulier et al. 2000; Rutschmann et al. 2000b; Lu et al. 2001). However, mutations in these genes do not induce susceptibility to fungal infections, demonstrating that the immune responses regulated by the Imd pathway are required to resist Gram-negative bacterial but not fungal infections (Leulier et al. 2000; Rutschmann et al. 2000b).

Some significant conclusions of recent studies on the regulation of Drosophila antimicrobial peptide gene expression are that the Toll and Imd pathways do not share any components and that each pathway regulates specific Rel proteins (Leulier et al. 2000; Rutschmann et al. 2000b; Khush et al. 2001; Lu et al. 2001). The only evi- dence of interactions between the two pathways is the observation that both pathways are required to fully induce some of the antimicrobial peptide genes (Lemaitre et al. 1996), suggesting that these genes respond to combinations of Rel proteins controlled by the two pathways. The influence of each pathway on the expression of each antimicrobial peptide gene is apparent in flies carrying mutations that affect either the Toll or the Imd pathway: Drosomycin is mainly controlled by the Toll pathway; Diptericin and Drosocin can be fully activated by the Imd pathway; and full Metchnikowin, Defensin, Cecropin A and Attacin activation requires both pathways (Lemaitre et al. 1996; Levashina et al. 1998). None of the antimicrobial peptide genes are induced in imd; Toll double mutant flies, demonstrating that Imd and Toll are two essential pathways that regulate antimicrobial gene expression pathways (Lemaitre et al. 1996).

Despite our increased understanding of the regulation of antimicrobial peptide gene expression in flies, various intermediates in the Toll and Imd pathways remain uncharacterized: For example, the kinase that targets Cactus for degradation in the Toll pathway and the receptoradaptor complex that regulates the Imd pathway have not been identified. Following the observations that null mutations affecting the Imd pathway are not required for viability (Hedengren et al. 1999; Leulier et al. 2000; Rutschmann et al. 2000b; Lu et al. 2001), we initiated a search for additional members of the Imd pathway by screening for nonlethal mutations that induce susceptibility to Gram-negative bacterial infection in adult flies. We now report that null mutations in the Drosophila transforming growth factor activated kinase 1 gene (dTAK1) encoding the Drosophila homolog of the mammalian mitogen-activated protein kinase kinase kinase (MAPKKK) TAK1 (Takatsu et al. 2000) induce high susceptibility to Gram-negative bacterial infection and block antibacterial peptide gene expression. Our results indicate that $d T A K 1$ codes for a new component of the Imd pathway.

\section{Results \\ D10 is required to resist Gram-negative bacterial infection}

To identify Drosophila genes that mediate defense reactions to bacterial infection, we tested 2500 lines carrying ethyl methanesulfonate (EMS)-induced mutations on the $\mathrm{X}$ chromosome for susceptibility to bacterial infection: we pricked male adult flies with a needle dipped into a pellet of the Gram-negative bacterial species Erwinia carotovora carotovora 15 (E. carotovora 15) (Basset et al. 2000) and screened for mutants that failed to survive infection. Using this assay, we isolated nine recessive, homozygous viable mutations that render flies highly susceptible to E. carotovora 15 infection: Less than $10 \%$ of the mutated flies survived $48 \mathrm{~h}$ postinfection, whereas more than $90 \%$ of the wild-type flies survived (data not shown). These nine mutations fall into two complementation groups: B118, which represents five of the mutations, and D10, which represents the 
other four mutations. The $B 118$ group corresponds to the caspase encoding gene Dredd that we described recently (Leulier et al. 2000), and in the present report we characterize D10, the second gene isolated in our screen.

To compare the D10 phenotype with the phenotypes generated by mutations in other genes that regulate Drosophila immune responses, we first assayed the susceptibility of D10 and other mutant lines to infection by four microorganisms: we pricked flies with the Gramnegative bacteria Escherichia coli (E. coli), the Grampositive bacteria Micrococcus luteus (M. luteus), or the fungus Aspergillus fumigatus, and we naturally infected flies with the entomopathogenic fungus Beauveria bassiana (B. bassiana) (Fig. 1). The D10 phenotype is similar to the imd and Relish phenotypes; flies carrying the D10, imd, and Relish mutations are susceptible to Gram-negative bacterial infection and resistant to Grampositive bacterial and fungal infections, although D10 flies, like imd flies, exhibit slightly lower susceptibility to Gram-negative bacterial infection compared to Relish mutants. In contrast, mutations in the spz gene render flies susceptible to fungal infections, and only flies carrying mutations in both $s p z$ and imd are susceptible to Gram-positive bacterial infection. This survival analysis demonstrates that the D10 gene product, like Imd and
Relish, is required to resist Gram-negative bacterial infection.

\section{D10 regulates antibacterial peptide gene expression}

The Toll pathway is required for the full induction of the antifungal peptide genes and a subset of the antibacterial peptide genes. Mutations that block the Toll pathway reduce the expression of these genes; conversely, mutations that block the Imd pathway reduce the expression of genes with antibacterial activity (for review, see Engstrom 1999; Khush et al. 2001). To determine how the D10 mutation affects antimicrobial peptide gene expression, we monitored the levels of Diptericin, Cecropin A, Defensin, and Attacin, which encode antibacterial peptides, Drosomycin, which encodes an antifungal peptide, and Metchnikowin, which encodes a peptide with both antibacterial and antifungal activity (Hoffmann and Reichhart 1997), in flies homozygous for two D10 alleles. In addition, we compared the $D 10$ phenotype with all of the previously identified mutations affecting the Imd pathway and with a $s p z$ mutation that blocks the Toll pathway.

Pricking adult flies with a mixture of Gram-positive and Gram-negative bacteria activates the expression of
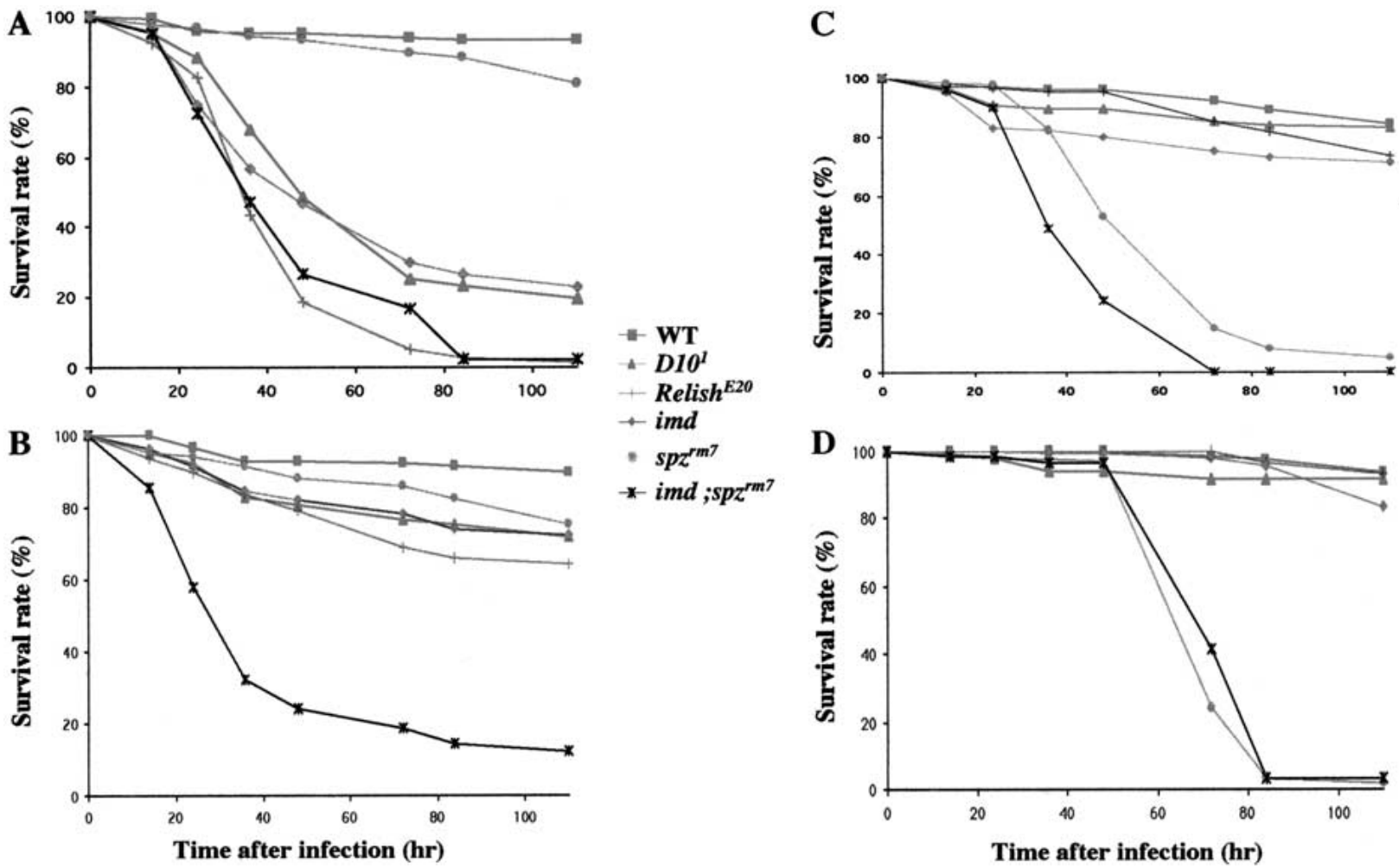

Figure 1. D10 is required to resist Gram-negative bacterial infection. The survival rates of wild-type $\left(\mathrm{Canton}^{\mathrm{S}}, \mathbf{\square}\right), \operatorname{imd}(\bullet), \operatorname{spz} z^{r m 7}(\mathbf{\bullet})$, $D 10^{1}(\mathbf{\Delta})$, Relish ${ }^{E 2 O}(+)$ and $i m d ; s p z^{r m 7}(*)$ flies after different types of infection are presented. From 100 to 200 adults, aged $2-4 \mathrm{~d}$, were pricked with a needle previously dipped into one of the following: E. coli $(A)$; M. luteus $(B)$, Aspergillus fumigatus (C), or naturally infected by $B$. bassiana $(D)$. The infected flies were incubated at $29^{\circ} \mathrm{C}$ and transferred to fresh vials every $3 \mathrm{~d}$. All of the different mutants flies tested exhibited more than $80 \%$ survival $100 \mathrm{~h}$ after challenge by a clean injury (data not shown). Mutations in $D 10$, Relish, and imd render adult flies highly susceptible to E. coli infection $(A)$. Only flies carrying both the imd and spz mutation are sensitive to $M$. luteus infection $(B)$. The spz gene is required to resist fungal infections $(C$ and $D)$. 
all the antimicrobial peptide genes; in the D10 mutants, however, mixed Gram-negative/Gram-positive infections induce significant levels of only Drosomycin and Metchnikowin (Fig. 2A,B). Quantitative measurements of three independent RNA blot experiments show that in D10 flies, Drosomycin is induced to wild-type levels, Metchnikowin is induced to $70 \%$ of wild-type levels, Cecropin A, Defensin, and Attacin are induced to $<25 \%$ of wild-type levels, and Diptericin is induced to $<5 \%$ of wild-type levels (Fig. 2B). This pattern of antimicrobial peptide gene expression in the D10 mutants is similar to the patterns displayed in mutants of the Imd pathway,

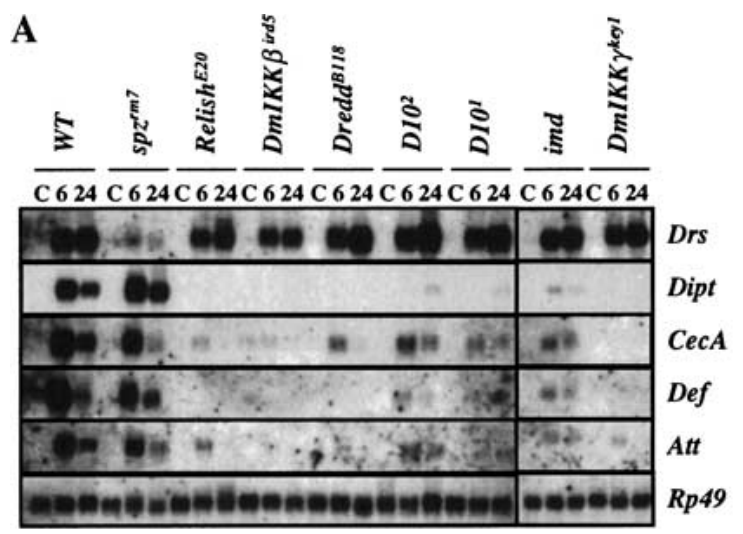

B

\begin{tabular}{|c|c|c|c|c|c|c|}
\hline \multirow{2}{*}{$\begin{array}{l}\text { Antimicrobial } \\
\text { peptides }\end{array}$} & \multicolumn{3}{|c|}{$W T$} & \multicolumn{3}{|c|}{$D^{\prime} 0^{\prime}$} \\
\hline & 0 & $6 h$ & $24 h$ & 0 & $6 \mathrm{~h}$ & $24 \mathrm{~h}$ \\
\hline Diptericin & $3 \pm 1$ & $100 \pm 0$ & $41 \pm 18$ & $0 \pm 0$ & $1 \pm 1$ & $5 \pm 3$ \\
\hline Cecropin A & $5 \pm 3$ & $100 \pm 0$ & $14 \pm 11$ & $1 \pm 1$ & $21 \pm 20$ & $12 \pm 12$ \\
\hline Attacin & $8 \pm 9$ & $100 \pm 0$ & $38 \pm 20$ & $3 \pm 3$ & $13 \pm 12$ & $10 \pm 7$ \\
\hline Defensin & $15 \pm 18$ & $100 \pm 0$ & $14 \pm 4$ & $1 \pm 1$ & $4 \pm 1$ & $7 \pm 5$ \\
\hline Metchnikowin & $19 \pm 27$ & $100 \pm 0$ & $110 \pm 15$ & $3 \pm 1$ & $71 \pm 52$ & $69 \pm 27$ \\
\hline Drosomycin & $12 \pm 6$ & $100 \pm 0$ & $123 \pm 35$ & $7 \pm 5$ & $95 \pm 42$ & $160 \pm 34$ \\
\hline
\end{tabular}

Figure 2. D10 regulates the expression of antibacterial peptide genes in adults. (A) A time course of antimicrobial gene expression in different mutant adults infected with a mixture of E.coli and $M$. luteus shows that D10, Dredd, Relish, DmIKK $\beta$, $D m I K K \gamma$, and imd predominantly control the expression of the antibacterial genes (Diptericin, Cecropin A, Attacin, and Defensin). The Northern blot was performed with total RNA extracted from wild-type, $D 10^{1}, D 10^{2}$, Dredd $^{B 118}$, imd, Relish ${ }^{E 20}$, $D m I K K \beta^{i r d 5}, D m I K K \gamma^{\text {key } 1}$ and $s p z^{r m 7}$ mutant adults at different time intervals after infection (indicated in hrs). After infection, flies were incubated at $25^{\circ} \mathrm{C}$. The blot was successively hybridized with the following cDNA probes: Diptericin (Dipt), Cecropin A (Cec A), Attacin (Att), Defensin (Def), Drosomycin (Drs) and Rp49. (C) Uninfected control flies. (B) The quantification of antimicrobial peptide gene expression in wild-type and $D 10^{1}$ mutant adults collected $6 \mathrm{~h}$ and $24 \mathrm{~h}$ after infection with a mixture of $E$. coli and $M$. luteus, expressed as the percentage of the induction seen in wild-type flies $6 \mathrm{~h}$ after infection. The signals from three independent Northern blot experiments were quantified with a Bio-Imager system, and the levels of immune gene expression were normalized with the corresponding value of the $R p 49$ signal. The average and standard deviation are shown. although the D10 mutations, like imd, have slightly weaker effects on antimicrobial peptide gene expression compared to the Dredd, DmIKK $\beta, D m I K K \gamma$, and Relish mutations (Fig. 2A) (Lemaitre et al. 1995; Hedengren et al. 1999; Leulier et al. 2000; Rutschmann et al. 2000b; Lu et al. 2001). This weaker phenotype in D10 and imd flies correlates well with their lower susceptibility to E. coli infection (Fig. 1).

\section{D10 does not function in the Toll pathway}

In contrast to the infections induced by pricking flies with mixtures of Gram-negative and Gram-positive bacteria, natural infections by the fungus $B$. bassiana selectively induce the expression of Drosomycin and Metchnikowin via the Toll pathway (Lemaitre et al. 1997). To determine whether D10 plays a role in the Toll pathway, we compared Drosomycin expression in different mutant backgrounds after fungal infection. We found that in contrast to mutations in $s p z$, mutations in D10 and components of the Imd pathway do not block Drosomycin induction (Fig. 3A); the variable Drosomycin levels in the different Imd pathway mutants are probably due to differing efficiencies of natural infection. Furthermore, mutations in D10 and Dredd do not block the constitutive Drosomycin expression induced by the dominant, gain-of-function Toll ${ }^{1 O B}$ mutation (Lemaitre et al. 1996) (Fig. 3B). The Toll and Imd pathways are the two major regulators of antimicrobial peptide gene expression in Drosophila, and flies carrying mutations in both the Toll and Imd pathways do not express any antimicrobial peptide genes (Lemaitre et al. 1996). Similarly, flies carrying $s p z$ in combination with either D10 or Dredd mutations fail to express any of the antimicrobial peptide genes after a mixed bacterial infection (Fig. 3C). We noted only a residual expression of the Drosomycin gene in the double mutant. The $D 10$ phenotypes that we have characterized, that is, susceptibility to Gram-negative bacterial infection, reduced induction of the antibacterial peptide genes, absence of detectable function in the Toll pathway, and a complete block of antimicrobial peptide gene expression in combination with a spz mutation, are identical to the phenotypes generated by mutations in members of the Imd pathway, suggesting that D10 encodes a novel component of the Imd pathway.

\section{D10 encodes the MAPKKK dTAK1}

Using susceptibility to Gram-negative bacterial infection as an assay, we first mapped $D 10$ by recombination to a region of the $\mathrm{X}$ chromosome distal to forked (data not shown). Deficiencies and additional genetic markers enabled us to further localize D10 to a portion of the cytogenetic interval 19E1 that, based on genomic annotation data (Consortium 1998), contains 25 genes (Fig. 4A). One of the 25 genes in this region is $d T A K 1$, which encodes a homolog of the mammalian TAK1 gene product (Takatsu et al. 2000). In mammals, TAK1 is implicated in the regulation of NF-кB as a component of the 
$\mathbf{A}$

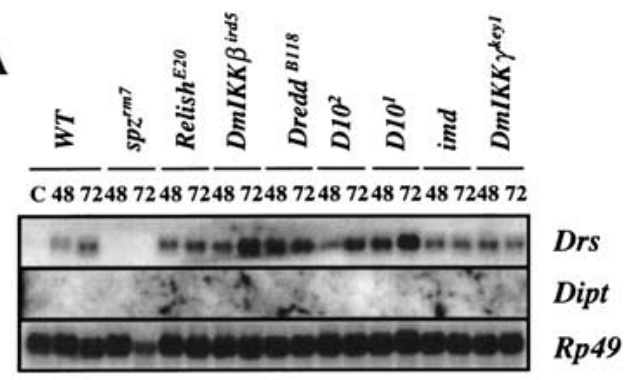

B
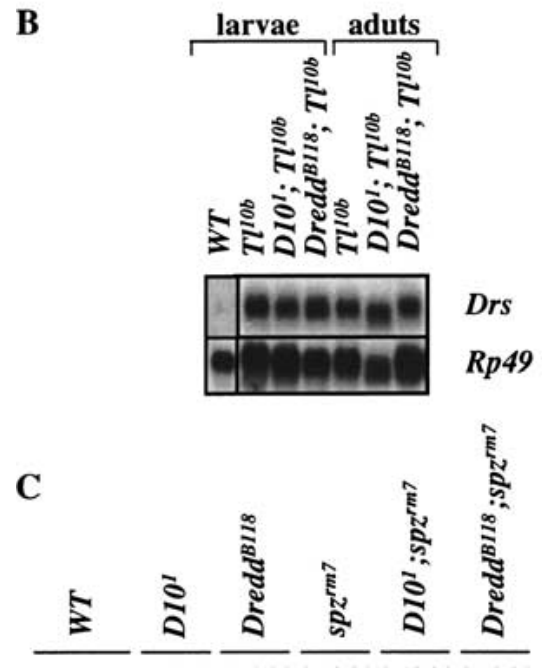

C 612246122461224612246122461224

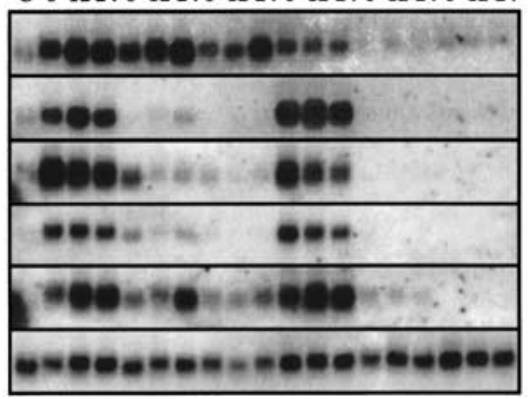

Drs

Dipt

$\operatorname{CecA}$

Att

Metch

$\operatorname{Rp} 49$

Figure 3. D10 mutants do not interfere with the Toll-mediated induction of Drosomycin expression. (A) Antimicrobial gene expression patterns in different mutant adults collected either $48 \mathrm{~h}$ or $72 \mathrm{~h}$ after natural infection by the entomopathogenic fungi $B$. bassiana shows that natural infection by $B$. bassiana induces a selective expression of Drosomycin that is dependent on $s p z$ but not on D10, Dredd, Relish, DmIKK $\beta, D m I K K \gamma$, and imd, indicating that the Toll-mediated expression of the Drosomycin gene by this fungus is not altered by mutations affecting the Imd pathway. The Northern blot was performed as described in Fig. 2. Flies naturally infected were incubated at $29^{\circ} \mathrm{C}$. The blot was successively hybridized with the following cDNA probes: Diptericin (Dipt), Drosomycin (Drs), and Rp49. (C) Uninfected control flies. (B) The high level of Drosomycin gene expression in uninfected $T 1^{10 b}, D 10^{1} ; \mathrm{Tl}^{10 \mathrm{~b}}$, and Dredd ${ }^{B 118}$; $T 1^{10 b}$ mutants demonstrates that D10 and Dredd are not required for the $\mathrm{Tl}^{10 \mathrm{~b}}$-driven constitutive expression of Drosomycin in larvae and adults. $(C) D 10^{1} ; s p z^{r m 7}$ and Dredd ${ }^{B 118} ; s p z^{r m 7}$ double mutants fail to express any antimicrobial peptide gene after mixed Gram-negative/Gram-positive bacterial infection, indicating that another pathway cannot substitute for the Toll and Imd pathways to induce antimicrobial peptide gene expression.
IL-1 signaling pathway, where it is thought to function upstream of the IKK complex (Sakurai et al. 1998; Ninomiya-Tsuji et al. 1999; Sakurai et al. 1999; Irie et al. 2000). Based on the established roles of an IKK complex and the NF-кB-like transactivator Relish in the Imd pathway, we selected $d T A K 1$ as a candidate for $D 10$.

We performed several different experiments to determine whether the D10 alleles correspond to mutations in $d T A K 1$. First, we tested the ability of a $15 \mathrm{~Kb}$ genomic fragment that contains $d T A K 1$ and annotation CG1812 (Fig. 4B) to rescue the immune response deficiency in D10 flies. Figures 4C and D show that D10 adults carrying the $d T A K 1$ transgene $\left(P\left[d T A K 1^{+}\right]\right)$both express Diptericin and resist Gram-negative bacterial infection at levels comparable to wild-type flies, demonstrating that this genomic fragment rescues the $D 10$ phenotypes. We also determined that overexpression of the $d T A K 1$ cDNA using the UAS/GAL4 system partially rescues Diptericin expression in the $D 10^{1}$ mutant (Fig. 6A, see below). We next sequenced the three $\mathrm{Kb}$ of $d T A K 1$ genomic coding sequence in our original $y, w$ strain and in each of the four homozygous D10 lines. The four D10 alleles all contain mutations within the $d T A K 1$ kinase domain, which led us to rename the $D 10$ alleles $d T A K 1^{1}$ to $d T A K 1^{4}: d T A K 1^{1}$ and $d T A K 1^{4}$ were generated by missense mutations in conserved residues, $d T A K 1^{2}$ was generated by a point mutation that creates a stop codon, and $d T A K 1^{3}$ contains a deletion of 31 base pairs that also results in a premature stop codon (Fig. 5A,B). All four dTAK1 alleles inhibit Diptericin induction by Gramnegative bacterial infection to the same degree (Fig. 4C), and this inhibition is not enhanced in flies heterozygous for each allele and a deficiency spanning $d T A K 1$ (data not shown). In addition, flies homozygous for the four alleles are equally susceptible to Gram-negative bacterial infection (data not shown). The apparent null phenotype manifested by the four $d T A K 1$ alleles indicates that the dTAK1 kinase domain is essential for dTAK1 function in the Imd pathway. This observation is supported by results from our experiments with a kinase dead form of dTAK1, dTAK1-K46R, which was previously reported to act as a dominant negative inhibitor of dTAK1 (Takatsu et al. 2000). dTAK1-K46R expression driven by the UAS/GAL4 system (Brand and Perrimon 1993) blocks Diptericin expression after mixed bacterial infection, confirming that dTAK1 is required for the Drosophila antibacterial immune response (Fig. 4C). The results of our rescue experiments, our sequencing data, and the dominant negative $d T A K 1$ mutant phenotypes together demonstrate that D10 encodes the MAPKKK dTAK1.

\section{dTAK1 functions upstream and Dredd functions downstream of the Drosophila IKK complex}

The loss-of-function $d T A K 1$ mutations that we isolated display immune response phenotypes that are very similar to the phenotypes generated by mutations in imd, $D m I K K \beta, D m I K K \gamma$, Dredd, and Relish, suggesting that these genes function together in the Imd pathway. Pre- 

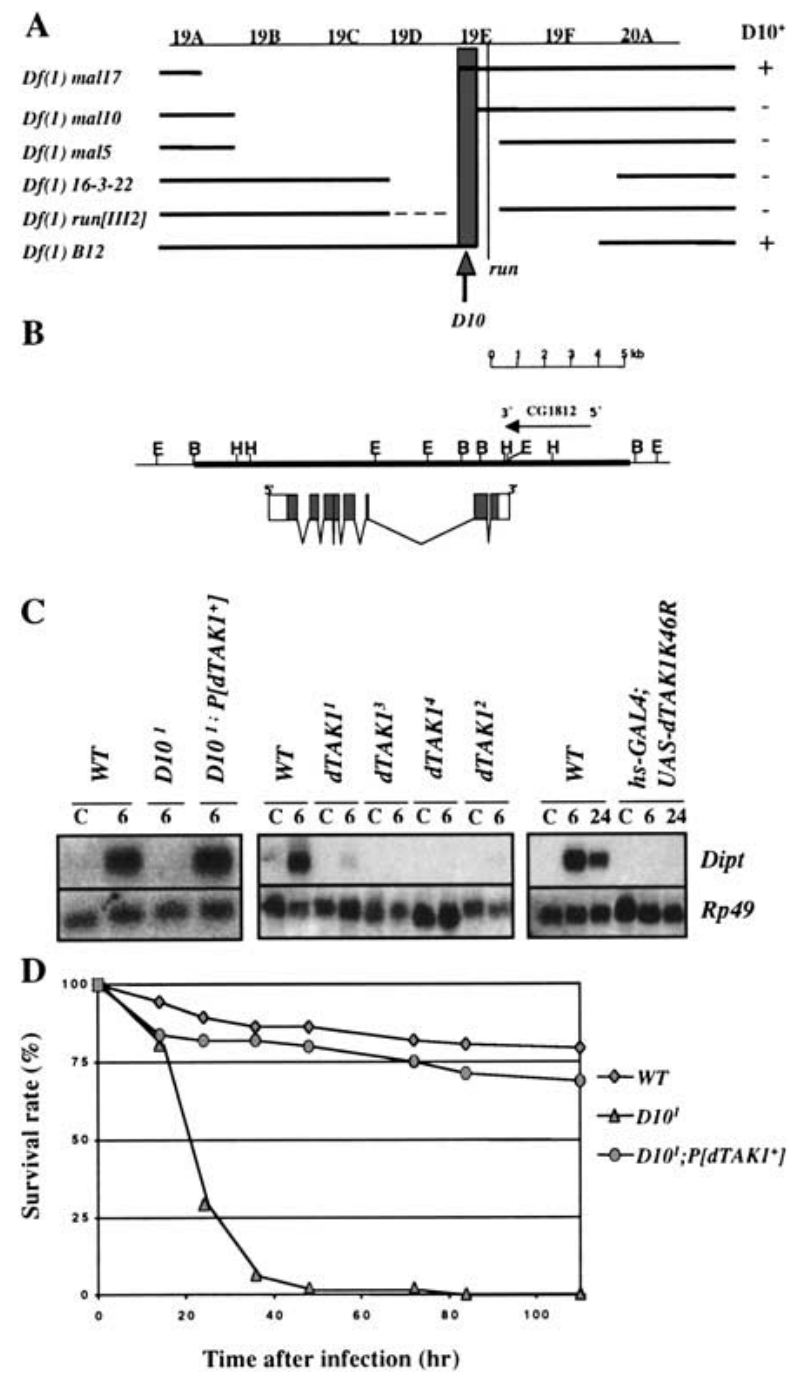

Figure 4. D10 encodes dTAK1. (A) Mapping the D10 region with deficiencies. A genetic map of the 19C-20A region of the $\mathrm{X}$ chromosome with the positions of the D10 gene and run. Deficiencies are indicated with their approximate breakpoints. The deficiencies that complement $D 10(+)$ or do not complement D10 (-) are indicated on the right. Resistance to E. carotovora 15 infection was used to assay for the presence of the D10 gene. According to these complementation tests, D10 maps to region $19 \mathrm{E} 1$ (as indicated by the shaded box). (B) A genomic map of the $d T A K 1$ locus showing the exon-intron organization of the gene and the $15 \mathrm{~kb}$ genomic DNA contained in the $P\left[d T A K 1^{+}\right]$transgene. Annotation CG1812 is indicated. (C) Northern blot analysis of total RNA extracted from adult flies infected with a mixture of Gram-positive (M. luteus) and Gram-negative (E. coli) bacteria shows that Diptericin expression is restored in $D 10^{1}$; $P\left[d T A K 1^{+}\right]$flies (left panel). The four alleles of $d T A K 1$ (1-4) completely block Diptericin induction after mixed bacterial infection (center panel). The overexpression of a dominant negative form of dTAK1 also blocks Diptericin induction in adult flies carrying both the hs-GAL4 and UAS-dTAK1 K46R transgenes collected $6 \mathrm{~h}$ and $24 \mathrm{~h}$ after bacterial challenge. A $1 \mathrm{~h}$ heat shock $\left(37^{\circ} \mathrm{C}\right)$ was performed just before infecting the flies. (C) Uninfected control flies. (D) D10 flies carrying the $P\left[d T A K 1^{+}\right]$ transgene resist $E$. carotovora 15 infection at levels comparable to wild-type flies. vious studies indicated that DmIKK $\gamma$ and DmIKK $\beta$ directly regulate Relish activity (Silverman et al. 2000); however, the positions of Imd, dTAK1, and Dredd in the Imd pathway were not determined. Double mutant analysis combining a Toll gain-of-function mutation that constitutively activates the Toll pathway and lossof-function mutations in other genes was a powerful tool for establishing the order of action of the genes encoding components of the Toll pathway (for review, see Belvin and Anderson 1996). Unfortunately, none of the mutations affecting the Imd pathway produce constitutive antibacterial immune responses; consequently, to avail of a genetic approach for ordering the Imd pathway, we overexpressed both Dredd and dTAK1 via the UAS/GAL4 system. In lines carrying a heat shock (hs)-GAL4 driver and either the Dredd or $d T A K 1$ cDNAs under the control of a UAS promoter, heat shock induces Diptericin expression to about $15-20 \%$ of the level observed in adults $6 \mathrm{~h}$ after bacterial infection (Fig. 6A). This result indicates that the overexpression of these two genes is
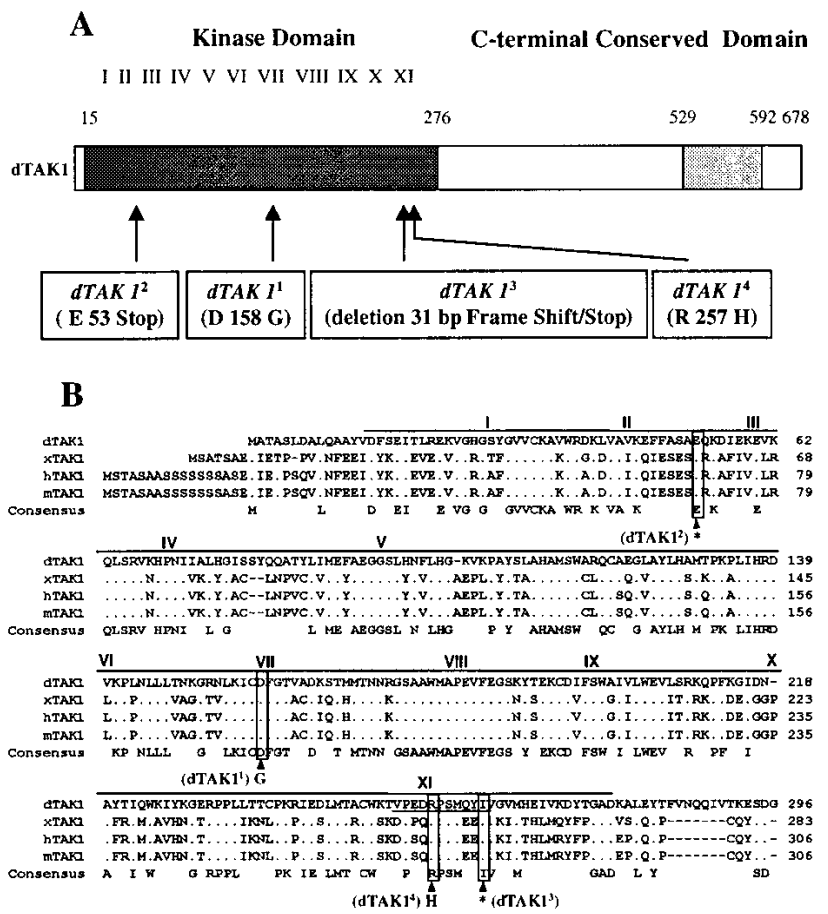

Figure 5. The $d T A K 1$ mutations affect the dTAK1 kinase domain. $(A)$ The four EMS-induced alleles of $d T A K 1$ each contain a single point mutation, or small deletion $\left(d T A K 1^{3}\right)$ that generate the indicated changes in the kinase region of the dTAK1 protein. (B) The amino acid sequence of the dTAK1 kinase domain is compared with that of Xenopus $(\mathrm{x})$ TAK1, one of three different forms of human (h) TAK1, and mouse (m) TAK1 (Takatsu et al. 2000). The exact positions of the four mutations in $d T A K 1$ are indicated. The $d T A K 1^{1}$ and $d T A K 1^{4}$ mutations induce amino acid substitutions in conserved residues of kinase domains 7 and 11 , respectively. The $d T A K 1^{2}$ and $d T A K 1^{3} \mathrm{mu}-$ tations generate stop codons $\left(^{*}\right)$ in the beginning or the end of the kinase domain. The stop codon in $d T A K 1^{3}$ is generated by a deletion of $31 \mathrm{bp}$ that produces a frameshift (the deleted codons are underlined). 


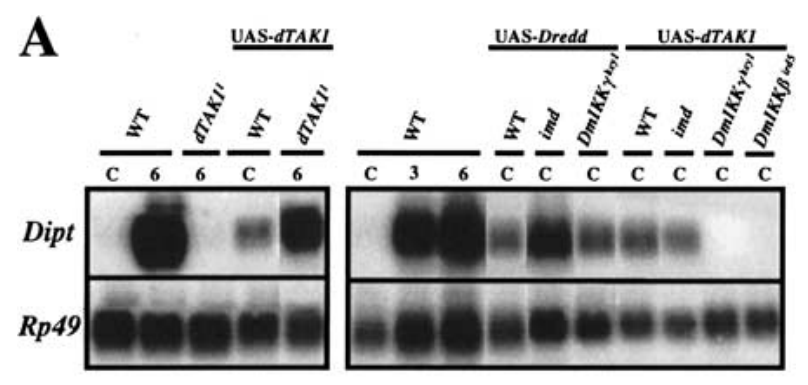

B

\begin{tabular}{|c|c|c|c|}
\hline Genotype & AS-Dredd & GAL4 Driver & B-gal. Units \\
\hline $\begin{array}{c}\text { WT } \\
\text { WT } \\
\text { WT } \\
\text { imd/imd } \\
\text { DmIKKK } \text { iss } / D m I K K \beta \text { ins } \\
\end{array}$ & $\begin{array}{l}- \\
\text { III } \\
\text { III } \\
\text { III } \\
\text { III }\end{array}$ & $\begin{array}{c}- \\
\text { yolk-GALA } \\
\text { yolk-GALA } \\
\text { yolk-GALA }\end{array}$ & $\begin{array}{c}0.2(0.3-20) \\
0.7(0.7-18) \\
49.5(19.9-59) \\
53.1(17.2-13) \\
44.7(4.5-4) \\
\end{array}$ \\
\hline $\begin{array}{c}\text { WT } \\
\text { Relish }^{230} / \text { Relish }\end{array}$ & $\begin{array}{l}\mathbf{I} \\
\mathbf{I}\end{array}$ & $\begin{array}{l}\text { yolk-GALA } \\
\text { yolk-GALA }\end{array}$ & $\begin{array}{c}70.9(11.3-3) \\
7.8(1.8-6) \\
\end{array}$ \\
\hline
\end{tabular}

Figure 6. A genetic analysis of the Imd pathway. (A) Overexpression of UAS-dTAK1 and UAS-Dredd (isoform $\delta^{\star}$ ) using the $U A S / G A L 4$ system was able to partially or completely rescue the $d T A K 1$ and Dredd mutations, respectively (dTAK1, left panel; Dredd, data not shown). Overexpression of these two constructs with an hs-GAL4 driver induces a weak but consistent expression of the Diptericin gene after heat shock in absence of infection (right panel). By classic genetic techniques, the UAS and GAL4 constructs were placed in the imd, $D m I K K \beta$, and DmIKK $\gamma$ genetic backgrounds. The right panel shows that the Diptericin expression induced by overexpressing $d T A K 1$ is blocked by the DmIKK $\beta$ and DmIKK $\gamma$ mutations but not by the imd mutation. In contrast, Dredd-mediated expression of Diptericin is not blocked in the DmIKK $\gamma$ and imd mutant backgrounds. Total RNA for Northern Blot analysis was extracted from adult flies collected $24 \mathrm{~h}$ after a $1 \mathrm{~h}$ heat shock $\left(37^{\circ} \mathrm{C}\right)$ that carry one copy of either a UAS-dTAK1 transgene (II chromosome) or a UAS-Dredd transgene (III chromosome) and one copy of the hs-GAL4 transgene (III chromosome). In the case of Dredd, we use a recombinant line carrying both UASDredd and hs-GAL4 on the same chromosome. (B) Overexpression of UAS-Dredd by the yolk-GAL4 driver induced a high level of Diptericin-lac $Z$ activity in absence of challenge that is dependent on Relish but not the imd or the DmIKK $\beta$ genes. Unchallenged female adult flies were generated that carry one copy of a Diptericin-lacZ ( $X$ chromosome) reporter gene in combination with one copy of a UAS-Dredd transgene ( $X$ or III chromosomes) and one copy of the yolk-GAL4 driver transgene (III chromosome) in wild-type, imd, DmIKKß, and Relish mutant backgrounds. The first column shows the genotype, and the second and third columns indicate the presence or absence (-) of the UAS-Dredd and yolk-GAL4 driver, respectively. The last column shows the level of Diptericin-lacZ expression monitored by LacZ titration (Manfruelli et al. 1999). Means for Diptericin-lac $Z$ expression are shown with standard deviation, and the number of repeats are given in brackets. The yolk-GAL4 driver expresses GAL4 specifically in the female adult fat body. The level of Diptericin-lacZ in 6 h-challenged females was 21 $(4.6 ; 26)$.

sufficient to activate the antibacterial pathway in the absence of infection. By using this UAS/GAL4 system to overexpress $d T A K 1$ and Dredd in various mutant back- grounds, we tested the epistatic relationships between dTAK1 and Dredd, and the other genes of the Imd pathway. Figure 6A shows that mutations in DmIKK $\beta$ and $D m I K K \gamma$, but not imd, block Diptericin induction by dTAK1 overexpression, indicating that dTAK1 functions downstream of Imd and upstream of the IKK complex. However, the Diptericin expression induced by Dredd overexpression was not affected by mutations in imd or $D m I K K \gamma$. For genetic reasons, we were unable to use Northern blot analysis to test the effect of mutations in Relish and DmIKK $\beta$, which are on the third chromosome, on the UAS-Dredd-induced Diptericin expression. Therefore, we overexpressed the UAS-Dredd transgene through a female, adult fat body driver (yolk-GAL4) and monitored Diptericin expression with a Diptericin-LacZ reporter gene (Fig. 6B). LacZ titration assays demonstrate that $\beta$-galactosidase activity is induced in lines overexpressing UAS-Dredd in the absence of infection. The Dredd-mediated Diptericin-LacZ induction was strongly reduced in Relish but not in DmIKK $\beta$ mutants, confirming our Northern blot results showing that Dredd functions downstream of the IKK complex and demonstrating that Dredd regulates Diptericin expression through Relish.

\section{Natural Gram-negative bacterial infection selectively activates the Imd pathway}

Natural fungal infections highlight the ability of Drosophila to discriminate between pathogens and activate specific immune response pathways that lead to adapted immune responses (for review, see Khush and Lemaitre 2000). Although only the Imd pathway is required to resist Gram-negative bacterial infection, the Toll pathway is still activated to some degree in flies pricked with Gram-negative bacteria (Lemaitre et al. 1997). This suggests that injury (and associated contamination) contributes to a nonspecific immune response. We recently demonstrated that E. carotovora 15 naturally infects the Drosophila larval gut and triggers both the local and systemic expression of antimicrobial peptide genes (Basset et al. 2000; Tzou et al. 2000). In contrast to infection by pricking, natural E. carotovora 15 infection induces Diptericin expression more strongly than Drosomycin expression (Basset et al. 2000). To compare the contribution of the Toll and Imd pathways to antimicrobial peptide induction after natural E. carotovora 15 infection, we quantified Diptericin and Drosomycin expression in larvae from various mutant lines after natural E. carotovora 15 infections. Figure 7 shows that the Diptericin expression induced by E. carotovora 15 natural infection is entirely dependent on the genes of the Imd pathway, confirming that Diptericin is exclusively regulated by the Imd pathway. Interestingly, the level of Diptericin induction was higher in $s p z$ mutants than in wild-type larvae. In addition, Drosomycin induction after E. carotovora 15 natural infection of larvae was also dependent on the Imd pathway: mutations blocking the Imd pathway have stronger affects than the spz mutation on Drosomycin expression induced by natural infection. These 


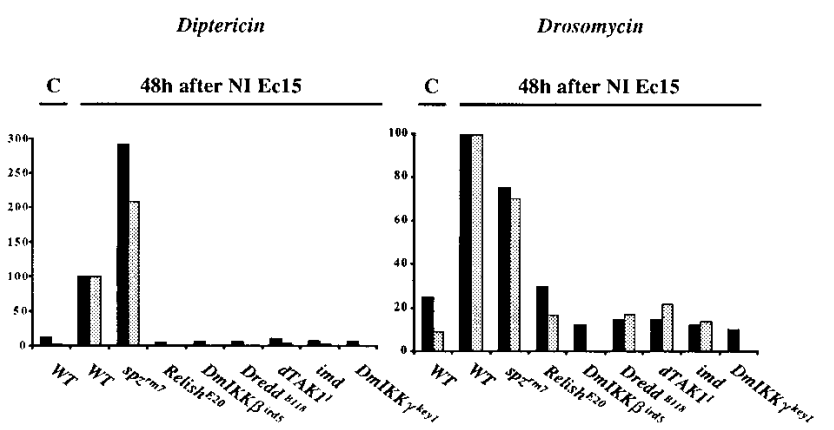

Figure 7. The Imd pathway regulates the expression of antimicrobial genes in larvae after infection by Gram-negative bacteria. The quantification of Diptericin and Drosomycin expression in wild-type and various mutant larvae collected $48 \mathrm{~h}$ after natural infection by E. carotovora 15 is expressed as the percentage of the induction seen in wild-type larvae. The signals from two independent Northern blot experiments were quantified with a Bio-Imager system, and the levels of antimicrobial peptide gene expression were normalized with the corresponding value of the $R p 49$ signal. Each bar represents the result of a single experiment. This results shows that $d T A K 1$, Dredd, Relish, $D m I K K \beta, D m I K K \gamma$, and imd control the expression of the antibacterial genes Diptericin and Drosomycin after natural infection by $E$. carotovora 15 . Larvae naturally infected by $E$. carotovora 15 were incubated at $29^{\circ} \mathrm{C}$.

data contrast somewhat with our previously published observation that the imd mutation did not block Drosomycin induction by $E$. carotovora 15 infection (Basset et al. 2000). However, our first analysis of Drosomycin induction by E. carotovora 15 was based on the qualitative analysis of a Drosomycin-lacZ reporter gene; we believe that the quantitative analysis of Drosomycin expression by Northern blot is a more accurate determination of Drosomycin expression patterns. In conclusion, our current results indicate that Drosomycin gene induction after natural Gram-negative bacterial infection is largely mediated by the Imd pathway.

Natural infections by E. carotovora 15 also trigger the local expression of antimicrobial peptide genes in various epithelial tissues, and both Diptericin expression in the anterior midgut and Drosomycin expression in the trachea are dependent on the imd gene (Ferrandon et al. 1998; Tzou et al. 2000). By assaying the expression of Diptericin-lacZ and Drosomycin-GFP reporter genes in naturally infected $d T A K 1$ and Dredd mutant larvae, we now show that both genes are required for Diptericin and Drosomycin expression in epithelial tissues after natural E. carotovora 15 infection (Fig. 8). These data confirm the predominant role of the Imd pathway in antimicrobial peptide regulation after natural E. carotovora 15 infection and suggest that Gram-negative bacterial recognition in flies preferentially activates the Imd pathway.

\section{Discussion}

The Drosophila homolog of TAK1 regulates antibacterial defense

Mammalian TAK1 was initially identified by a complementation assay in yeast for its ability to substitute for the MAPKKK Ste11p in the yeast MAPK pheromone pathway (Yamaguchi et al. 1995). TAK1 is a MAPKKK and contains a protein kinase domain in its $\mathrm{N}$-terminal region that is $\sim 30 \%$ identical to the catalytic domains of RAF1 and MEKK1. In mammals, TAK1 function has been extensively studied in transient transfection assays using cultured cells (for review, see Behrens 2000). These studies indicate that TAK1 can function in a signal transduction pathway that is triggered by the TGF $\beta$ superfamily of ligands. In addition, the overexpression of Xenopus TAK1 and an upstream activator called TAB1 induce embryonic ventralization. Studies of dominant negative mutant forms of TAK1 place TAK1 activity downstream of the BMP2/4 receptors (Shibuya et al. 1998).

Besides TGF $\beta$ signaling, TAK1 appears to be involved in various other signal transduction pathways. Cell culture experiments indicate that ceramide stimulates the TAK1 kinase activity and that ceramide-induced JNK/ SAPK activation is blocked by a dominant negative TAK1 mutant (Shirakabe et al. 1997). Biochemical studies also show that MAPKK4 and MAPKK3/MAPKK6 can be phosphorylated by TAK1, suggesting that TAK1 can activate the JNK pathway and/or the P38 MAPK pathways (Moriguchi et al. 1996; Wang et al. 1997), and other experiments demonstrate interactions between TAK1 and the Wnt signaling pathway (Ishitani et al. 1999). Several studies have pointed toward a role for TAK1 in the IL1-R/NF-kB signaling pathway (Sakurai et al. 1998; Ninomiya-Tsuji et al. 1999; Sakurai et al. 1999|. Dominant negative TAK1 mutants prevent NF-кB activation by IL1 or the TLRs (Ninomiya-Tsuji et al. 1999; Irie et al. 2000), and TAK1 appears to activate NF-кB by stimulating the ІкB kinase complex (IKK) that induces ІкB degra-

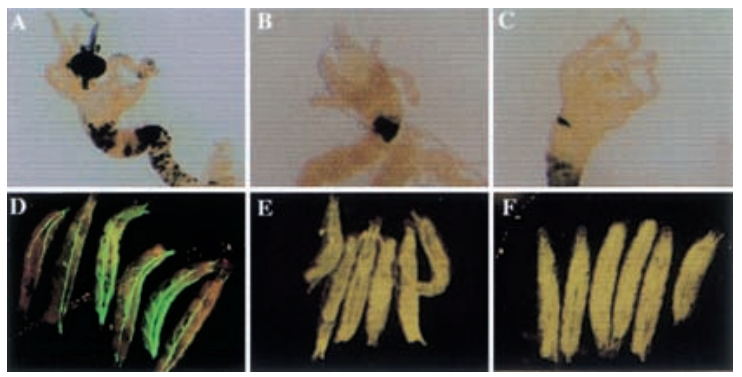

Figure 8. The Imd pathway regulates the local expression of Diptericin and Drosomycin in larvae after infection by Gramnegative bacteria. $(A-C)$. The expression of the Diptericin-lacZ reporter gene in the midgut after natural infection by E. carotovora 15 is blocked in $d T A K 1^{1}$ and Dredd ${ }^{B 118}$ mutants. Wildtype $(A), d T A K 1^{1}(B)$, and Dredd ${ }^{B 118}(C)$ mutant larvae carrying the Diptericin-1ac $Z$ reporter gene were collected $24 \mathrm{~h}$ after natural infection by E. carotovora 15 at $29^{\circ} \mathrm{C}$ and stained for lacZ activity as described (Tzou et al. 2000). (D-F). The expression of the Drosomycin-GFP reporter gene in trachea after natural infection by E. carotovora 15 is blocked in $d T A K 1^{1}$ and Dredd ${ }^{B 118}$ mutants. Wild-type $(D), d T A K 1(E)$, and $\operatorname{Dredd}^{B 118}(F)$ larvae carrying the Drosomycin-GFP reporter gene were collected $5 \mathrm{~d}$ after natural infection by $E$. carotovora 15 at $18^{\circ} \mathrm{C}$ and directly observed under an epifluoresence microscope. 
Vidal et al.

dation by the proteasome (Ninomiya-Tsuji et al. 1999; Sakurai et al. 1999). Furthermore, TAK1 interacts with TRAF6, which is a downstream effector of the IL1 receptor, suggesting that TAK1 links TRAF6 to the NIK-IKK cascade in the IL1-R/TLR signaling pathways (Ninomiya-Tsuji et al. 1999).

A Drosophila homolog of TAK1, dTAK1, that possesses an extensively conserved $\mathrm{NH} 2$-terminal kinase and a partially conserved C-terminal domain was identified recently (Takatsu et al. 2000). Ectopic dTAK1 expression induces apoptosis, whereas overexpression of a dominant negative form of dTAK1 during development results in various cuticle defects, some of which are similar to those observed in mutants in JNK signaling. This preliminary analysis suggested a role for $d T A K 1$ in the regulation of apoptosis and JNK signaling in flies (Takatsu et al. 2000; Mihaly et al. 2001). In the present study, we isolated four null mutations in $d T A K 1$. Each of these mutations affects the kinase domain of dTAK1, generating either a premature stop codon or an amino acid substitution. Flies deficient for $d T A K 1$ are highly susceptible to Gram-negative bacterial infection and fail to express antibacterial peptide-encoding genes after infection. This $d T A K 1$ phenotype is identical to the phenotype induced by mutations affecting components of the Imd pathway, suggesting that dTAK1 is a new component of the Imd pathway. dTAK1 does not, however, function in the Toll pathway that mediates dorsal-ventral patterning and antifungal immune responses in flies; the $d T A K 1$ mutations do not affect the Toll-mediated Drosomycin induction by fungal infection or the constitutive Drosomycin expression in Toll gain-of-function mutants, and the $d T A K 1$ mutants are not susceptible to fungal infection. Surprisingly, our results indicate that, in contrast to TGF $\beta$ - or JNK signaling-defective mutants, dTAK1 flies are viable and do not display obvious developmental defects. The developmental phenotypes generated by overexpressing a dominant negative form of TAK1 (Takatsu et al. 2000; Mihaly et al. 2001) were not observed in the $d T A K 1$-deficient mutants, although we have shown that the dominant negative dTAK1 mutant does phenocopy the $d T A K 1$ mutant immune phenotype. Our results demonstrate that dTAK1 is either not involved in JNK and TGF $\beta$ signaling in Drosophila or that its function in these pathways is redundant with another MAPKKK.

\section{Ordering the Imd pathway}

Overexpressing $d T A K 1$ or Dredd via the UAS/GAL4 system leads to weak but consistent Diptericin expression in the absence of infection, indicating that overexpressing these two genes is sufficient to turn on the antibacterial pathway. The constitutive Diptericin expression in the $d T A K 1$ and Dredd overexpression lines also allowed us to analyze the epistatic relationship between $d T A K 1$, Dredd, and the other recently identified regulators of antibacterial defense, and, in agreement with their similar mutant phenotypes, our results confirm that Imd, dTAK1, the DmIKK complex, and Dredd func- tion in a pathway that culminates in Relish activation (Fig. 9). Our data suggest a model in which Imd functions upstream of dTAK1 and is the most upstream component currently identified in the Imd pathway. The imd gene was recently cloned and encodes a death domaincontaining protein with homology to the mammalian RIP (P. Georgel et al., in prep.). In mammals, RIP appears to function in an adaptor complex associated with the tumor necrosis factor (TNF) receptor, and our genetic placement of imd suggests that IMD has a conserved function in flies as part of a receptor-adaptor complex that responds to Gram-negative bacterial infection (Fig. 9).

Our epistatic analysis also indicates that dTAK1 functions upstream of the DmIKK complex. In mammals, TAK1 functions upstream of the IKK complex, and it is not yet clear whether TAK1 directly activates the IKK

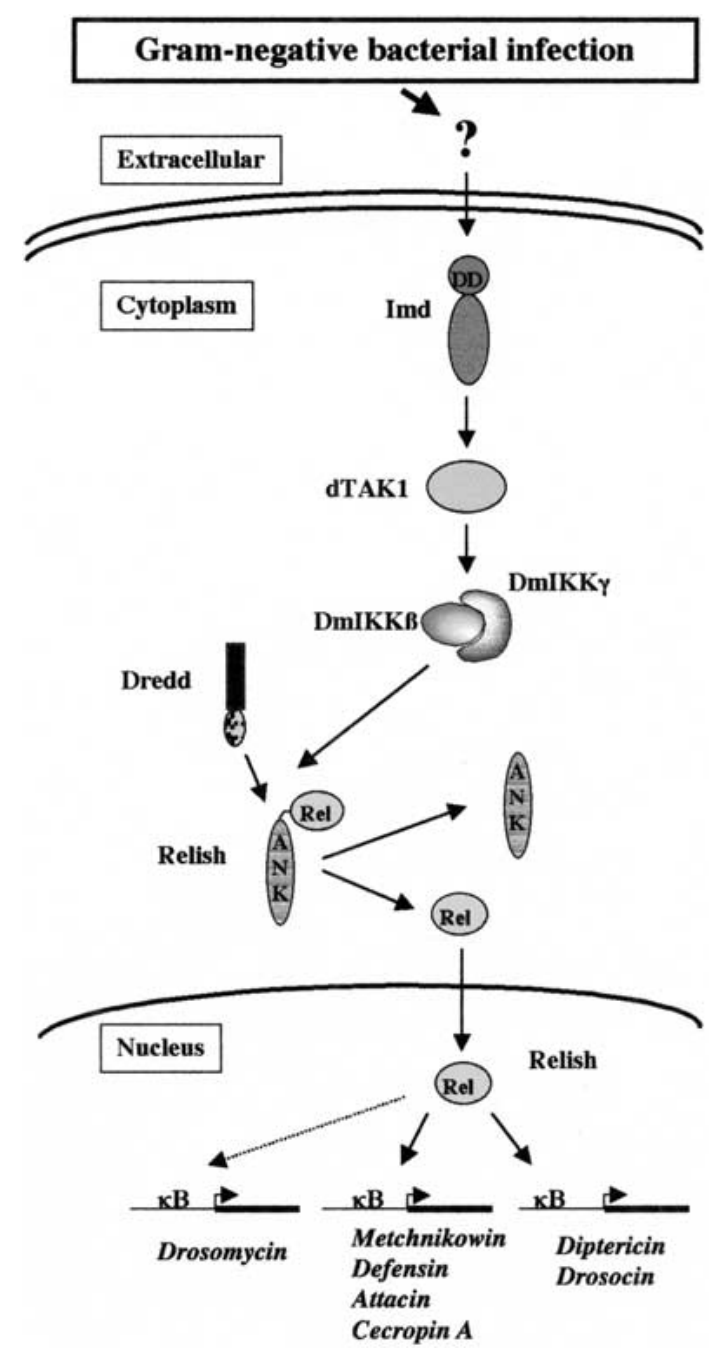

Figure 9. The Imd pathway. A model of the Imd pathway that is based on our epistatic studies shows that dTAK1 functions upstream and Dredd functions downstream of the DmIKK complex. Previous studies have shown that Relish is activated by the DmIKK complex (Silverman et al. 2000). See Discussion for additional details. 
complex (Sakurai et al. 1999) or whether TAK1 functions through NIK, another MAPKKK that is involved in NF$\kappa \mathrm{B}$ regulation (Ninomiya-Tsuji et al. 1999). A strong NIK homolog is not apparent in the Drosophila genome (Khush and Lemaitre 2000), suggesting that dTAK1 may directly activate the DmIKK complex after infection. dTAK1 flies have slightly weaker phenotypes than $D m I K K \beta, D m I K K \gamma$, Dredd, and Relish mutants, and this may correlate with the function of dTAK1 at an early stage of the antibacterial cascade where multiple inputs, some of which bypass dTAK1, regulate the DmIKK complex.

Dredd encodes a caspase with a large prodomain and is related to mammalian Caspase 8 (Rodriguez et al. 1999). Our genetic analysis indicates that Dredd functions downstream of the DmIKK complex and induces Diptericin expression through Relish, which confirms previous observations by Stöven et al. (2000), who showed that after infection Relish is rapidly cleaved by a proteasome-independent mechanism and that this cleavage is blocked in Dredd mutants. There is increasing evidence that caspases are involved in physiological processes other than apoptosis, and Dredd function downstream of the DmIKK complex suggests that Dredd participates directly, or indirectly via an effector caspase, in Relish cleavage. In mammals, apical caspases are activated by the processing of a prodomain, and a future issue to address is whether Dredd is constitutively active or is regulated by the DmIKK complex or another upstream component of the Imd pathway.

\section{Independent activation of the Toll and Imd pathways}

A current view of the antimicrobial response in Drosophila is that the induction of the antimicrobial peptides are controlled by two distinct pathways, Toll and Imd, that each regulate specific rel/NF-кB proteins (Leulier et al. 2000; Rutschmann et al. 2000b; Khush et al. 2001). These pathways are the two main immunoresponsive signaling cascades, as illustrated by our observation that flies carrying a mutation in both pathways fail to express any antimicrobial peptide genes and are highly susceptible to infection by all types of microorganisms. The ability to differentiate between microbes and activate each of these pathways independently, in response to different types of infection, appears to be one mechanism for mounting adapted immune responses. For example, the specific induction of Drosomycin after natural infection by the entomopathogenic fungus $B$. bassiana is due to the selective activation of the Toll pathway since, as indicated by our present results, this induction is not affected by mutations that block the Imd pathway. Natural infection by the Gram-negative bacterial species E. carotovora 15 leads to a predominant expression of antibacterial rather than antifungal peptide genes, and reveals the ability of Drosophila to mount adapted innate immune responses to Gram-negative bacterial infection (Basset et al. 2000). Our present findings indicate that antimicrobial peptide gene expression after natural
E. carotovora 15 infections is largely mediated through the Imd pathway, and that both Diptericin and Drosomycin expression after E. carotovora 15 infection are impaired in mutants affecting components of the Imd pathway. The high level of Diptericin expression and the low level of Drosomycin expression observed in this context probably reflect the differential responses of these two target genes to the selective activation of the Imd pathway. In the trachea, a major site of infection in insects, natural infection by E. carotovora 15 triggers Drosomycin gene expression in an Imd-dependent manner (Tzou et al. 2000; present finding). This illustrates the ability of the Drosomycin gene to be weakly turned on in response to Gram-negative bacterial infection by the Imd pathway, independently of any Toll input.

\section{Interactions between the Toll and Imd pathways}

Except for Diptericin and Drosocin, all of the antimicrobial peptide genes are regulated by inputs from both the Toll and Imd pathways; some, like Defensin, require the concomitant activation of both pathways, while others, such as Drosomycin and Metchnikowin, can be induced independently by each of these pathways (Lemaitre et al. 1996; Levashina et al. 1998). One hypothesis is that the differential regulation of antimicrobial peptide genes reflects their sensitivity to specific combinations of the Rel proteins that are the end targets of the Toll and Imd pathways (Han and Ip 1999). For example, Diptericin induction after infection is fully dependent on Relish and is not altered in mutants deficient for both Dif and Dorsal activity (Hedengren et al. 1999; Manfruelli et al. 1999; Meng et al. 1999). In agreement, Diptericin gene expression is almost completely abolished in mutants deficient in Imd signaling. Interestingly, we observed that the level of Diptericin gene expression is higher in $s p z$ mutant larvae than in wild-type larvae after natural Gram-negative bacterial infection (Fig. 7), and we observed a similar response after mixed Gram-negative/ Gram-positive bacterial infection in adult flies (Fig. 2A). These observations are consistent with our previous studies of Diptericin expression in spz mutants, although those results were not highlighted earlier (Fig. 2 in Lemaitre et al. 1996; Fig. 2A in Leulier et al. 2000). The effect of the $s p z$ mutation on Diptericin induction points to a negative effect of the Toll pathway on this gene. Our hypothesis is that Relish homodimers are the most potent activators of Diptericin, and that by blocking Dif and Dorsal activation, mutations in the Toll pathway may increase the formation of Relish homodimers versus Relish-Dif or Relish-Dorsal heterodimers. Together these observations indicate how the differential activation of these two pathways leads in a subtle way to the preferential expression of specific target genes.

\section{Concluding remarks}

We describe here the identification of null mutations in the Drosophila homolog of TAK1. Our genetic analysis 
shows that dTAK1, like the other components of the Imd pathway, is required for the induction of antibacterial peptide gene expression, and dTAK1's role in the Drosophila immune response is highlighted by the strong susceptibility of $d T A K 1$ mutants to Gram-negative bacterial infection. In mammals, experiments with tissue cultured cells indicate a role for TAK 1 in the IL1-R/TLRNF-кB pathway that regulates innate immune response. These data, and the results of our genetic studies of flies, suggest that the role of this MAPKKK in the control of the rel/NF- $\kappa \mathrm{B}$ innate immune response has been conserved during evolution, thereby strengthening the parallels between the regulation of mammalian and Drosophila innate immune responses. The list of common factors used by flies and mammals to regulate rel/NF- $\mathrm{B}$ proteins has increased in the last few years, but as shown for dTAK1 here, it appears that the regulators that function in the IL1/TLR pathway in mammals function independently in either the Toll or Imd in Drosophila. The existence of two distinct pathways for regulating NF-кB in flies may allow for the production of specific subsets of antimicrobial peptides against different pathogens (Khush et al. 2001).

We identified $d T A K 1$ in a screen for X-linked genes in Drosophila that are required to resist Gram-negative bacterial injection. Our findings illustrate the power of Drosophila genetics, since survival against challenge is a simple screen for genes involved in the Drosophila immune response. However, it is striking that among the 2500 mutagenized lines that we tested for susceptibility to Gram-negative bacterial infection, only mutations in Dredd and $d T A K 1$, two components of the Imd pathway, were identified. This result suggests that the Imd pathway controls the most important mechanisms for combating Gram-negative bacterial infection in flies.

\section{Materials and methods}

\section{Drosophila strains}

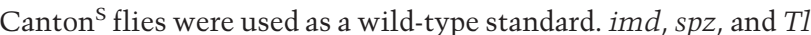
alleles are described elsewhere (Lemaitre et al. 1996). Relish ${ }^{E 20}$, $D_{\text {Dredd }}{ }^{B 118}, D m I K K \beta^{\text {ird } 5}\left(\right.$ ird $\left.5^{1}\right)$, and $D m I K K \gamma^{\text {key } 1}\left(\right.$ kenny $\left.^{1}\right)$ are either strong or null alleles of Relish, Dredd, DmIKK $\beta$, and $D m I K K \gamma$ (Hedengren et al. 1999; Leulier et al. 2000; Rutschmann et al. 2000b; Lu et al. 2001). $\operatorname{dTAK}^{1}\left(D 10^{1}\right), d T A K 1^{2}$ $\left(D 10^{2}\right), d T A K 1^{3}$, and $d T A K 1^{4}$ are four EMS-induced mutations of $d T A K 1$ that were generated in a yw chromosome. Diptericin$l a c Z$ is a $P$ transgene containing a fusion between $2.2 \mathrm{~kb}$ of upstream sequence from the Diptericin gene and the coding sequences from the $\beta$-galactosidase gene (Tzou et al. 2000). Drosomycin-GFP is a $P$ transgene containing a fusion between 2.4 $\mathrm{kb}$ of upstream sequence from the Drosomycin gene and the coding sequences from the Green Fluorescent Protein gene (Ferrandon et al. 1998). The precise fly genotypes are: imd: $b r, p r$, imd; $s p z^{r m 7}: s p z^{r m 7} / s p z^{r m 7}$; Relish: Relish ${ }^{E 20}, e_{;} \operatorname{Dredd}^{B 118}: y, w$, Dredd ${ }^{B 118}$; dTAK1: $y, w, d T A K 1 ; D m I K K \beta^{\text {ird5 }}: D \operatorname{DmIKK} \beta^{\text {ird5 }}, c a$, Diptericin-lacZ; DmIKK $\gamma^{\text {key1 }}:$ y,w, Diptericin-lacZ, Drosomy$c i n-G F P ; c n, b w, s p, D m I K K \gamma^{k e y 1}$. For survival studies, we used a Relish ${ }^{E 2 O}$ stock from which the ebony mutation was removed, because ebony affects survival levels (Lemaitre et al. 1996). Drosophila stocks were maintained at $25^{\circ} \mathrm{C}$. After infection, flies were incubated at either $25^{\circ} \mathrm{C}$ for Northern blots or $29^{\circ} \mathrm{C}$ for survival assays.

\section{Mutagenesis}

$y, w$ male flies were treated with 25 mM EMS. The mutagenized males were crossed with females $C(1) D X y, w, f$, and single $\mathrm{F}_{1}$ males were back-crossed to the same female stock to establish independent lines. Six $F_{2}$ males from each line were pricked with a needle dipped in a concentrated pellet of E. carotovora 15 $(\mathrm{OD}=200)$, and incubated at $29^{\circ} \mathrm{C}$ overnight. Lines that showed more than four dead flies out of six were retested. Canton ${ }^{\mathrm{S}}$ and imd flies were used as internal controls. For each selected line, a homozygous stock was established by crossing $\mathrm{F}_{2}$ males with FM3/1(1)44ter females. Survival after Gram-negative bacterial infection was sufficiently reliable to establish complementation groups. Initial mapping was performed by monitoring resistance to $E$. carotovora 15 infection in recombinants between the mutated chromosome and a chromosome carrying the $y^{+}, c v, f$ markers. Additional mapping was done with the $\mathrm{X}$ chromosome deficiency kit from the Bloomington stock center.

\section{Infection experiments}

Bacterial infections were performed by pricking third instar larva or adults with a thin needle previously dipped into a concentrated culture (O.D 200) of E.coli, M. luteus, or a mixture of the two bacteria. Natural infections with $B$. bassiana were performed by shaking anesthetized flies for thirty seconds in a Petri dish containing a sporulating fungal culture (Lemaitre et al. 1997). Bacterial and fungal strains were previously described (Lemaitre et al. 1997). Natural infection by E. carotovora 15 were performed by incubating Drosophila larvae in a mixture of crushed banana and bacteria (Basset et al. 2000).

\section{Overexpression studies}

$U A S-d T A K 1$ and UAS-dTAK1-K46R have been described previously (Takatsu et al. 2000). UAS-dTAK1-K46R encodes a dominant negative form of dTAK1 in which lysine 46 was replaced by an arginine. The full-length Dredd isoform $\delta^{\star}$ cDNA was cloned as a XhoI-BamH1 fragment from $p M T$-Dredd $\delta^{*}-M y c$ (Rodriguez et al. 1999) into XhoI-BamH1 digested $p U A S T$ vector. $W^{1118}$ flies were transformed using $P$ element-mediated transformation. The yolk-GAL4 driver strongly expresses GAL4 in the female adult fat body (J.M. Reichhart, pers. comm.). The hsp70-GAL14 driver expresses GAL4 ubiquitously after heat shock.

\section{Northern blot analysis}

Total RNA extraction and Northern blotting experiments were performed as described (Lemaitre et al. 1997).

\section{Sequencing of dTAK1 alleles}

Genomic DNA from the $y, w$ strain and the four dTAK1 mutants was extracted from single adult flies as described (Gloor and Engels 1992). One microliter of genomic DNA was used for each PCR amplification. Specific oligonucleotide primers for the $d T A K 1$ gene were synthesized and used to amplify overlapping fragments of the $d T A K 1$ coding sequence. The resulting single-fragment PCR products were purified using a Qiagen purification column and sequenced using the BigDye Terminator Cycle sequencing ready reaction (PE Applied Biosystems). The sequence profiles were analyzed on Edit View 1.0.1 ABI Prism 
(PE Applied Biosystems) and compared to the dTAK1 cDNA sequence published previously.

\section{Cloning and transformation of $\mathrm{P}[\mathrm{dTAK} 1]$}

A P1 Drosophila filter (Genome Systems) was hybridized with a ${ }^{32} \mathrm{P}$-labeled $3.5 \mathrm{~kb} d T A K 1 \mathrm{cDNA}$ probe. Three clones were isolated, and one of them (DS08905) was used for the following experiments. The $d T A K 1$ coding region $(10.0 \mathrm{~kb}$ and $5.8 \mathrm{~kb}$ BamH1 fragments and a $2.6 \mathrm{~kb}$ EcoRI fragment) was subcloned into pBlueScriptII KS+ and sequenced using synthetic sequence primers. Drosophila Genome Project sequencing data, AC014558, (BDGP) was also used to confirm the genomic sequencing. A $15 \mathrm{~kb}$ genomic fragment that contains $d T A K 1$ coding region as well as $2.5 \mathrm{~kb} 5^{\prime}$ - and $4.8 \mathrm{~kb} 3^{\prime}$-untranslated regions were amplified by PCR with Takara LA Taq (Takara Biomedical) by using PCR primers. This genomic fragment was digested with XhoI and NotI and then subcloned into the Drosophila transformation vector $\mathrm{P}\{\mathrm{CaSpeR}-4\}$. Flies bearing transgenes were generated by general P-element mediated transformation.

\section{Acknowledgments}

We thank Kathryn Anderson, Dominique Ferrandon, and Anthony Rodriguez for providing fly stocks or plasmids and our colleagues Isaline Rowe, Stéphanie Gobin, Brigitte Maroni, Thomas Ranson, Ennio de Gregorio, and Pedro Santamaria for assistance and stimulating discussions. We are especially grateful to Jean Marc Reichhart for providing the yolk-Gal4 line prior to publication. S.V. was supported by Praxis XXI/BD/21915/99 (Portugal). M.N. thanks Dr. N. Ueno for providing laboratory space and supplies as well as valuable advice and Dr. H. Takatsu for his contribution to an earlier stage of the work. This project was funded by ATIPE CNRS, the Fondation pour la Recherche Médicale (FRM) and Programme Microbiologie (PRMMIP98).

The publication costs of this article were defrayed in part by payment of page charges. This article must therefore be hereby marked "advertisement" in accordance with 18 USC section 1734 solely to indicate this fact.

\section{References}

Aderem, A. and Ulevitch, R.J. 2000. Toll-like receptors in the induction of the innate immune response. Nature 406: 782787.

Anderson, K.V. 2000. Toll signaling pathways in the innate immune response. Curr. Opin. Immunol. 12: 13-19.

Basset, A., Khush, R., Braun, A., Gardan, L., Boccard, F., Hoffmann, J., and Lemaitre, B. 2000. The phytopathogenic bacteria, Erwinia carotovora, infects Drosophila and activates an immune response. Proc. Nat1. Acad. Sci. 97: 3376-3381.

Behrens, J. 2000. Cross-regulation of the Wnt signalling pathway: A role of MAP kinases J. Cell Sci. 113: 911-919.

Belvin, M.P. and Anderson, K.V. 1996. A conserved signaling pathway: The Drosophila toll-dorsal pathway Annu. Rev. Cell Dev. Biol. 12: 393-416.

Brand, A.H. and Perrimon, N. 1993. Targeted gene expression as a mean of altering cell fates and generating dominant phenotypes. Development 118: 401-415.

Consortium, T.F. 1998. FlyBase-A Drosophila database. Nucleic Acids Res. 26: 85-88.

Elrod-Erickson, M., Mishra, S., and Schneider, D. 2000. Interactions between the cellular and humoral immune responses in Drosophila. Curr. Biol. 10: 781-784.

Engstrom, Y. 1999. Induction and regulation of antimicrobial peptides in Drosophila. Dev. Comp. Immunol. 23: 345-358.
Ferrandon, D., Jung, A.C., Criqui, M., Lemaitre, B., UttenweilerJoseph, S., Michaut, L., Reichhart, J., and Hoffmann, J.A. 1998. A Drosomycin-GFP reporter transgene reveals a local immune response in Drosophila that is not dependent on the Toll pathway. EMBO J. 17: 1217-1227.

Gloor, G.B. and Engels, W.R. 1992. Single-fly DNA preps for PCR. Drosoph. Inf. Serv. 75: 148-149.

Han, Z.S. and Ip, Y.T. 1999. Interaction and specificity of Relrelated proteins in regulating Drosophila immunity gene expression. J. Biol. Chem. 274: 21355-21361.

Hedengren, M., Asling, B., Dushay, M.S., Ando, I., Ekengren, S., Wihlborg, M., and Hultmark, D. 1999. Relish, a central factor in the control of humoral but not cellular immunity in Drosophila. Mol. Cell. 4: 827-837.

Hoffmann, J. and Reichhart, J. 1997. Drosophila immunity. Trends Cell. Biol. 7: 309-316.

Hoffmann, J.A., Kafatos, F.C., Janeway, C.A., and Ezekowitz, R.A. 1999. Phylogenetic perspectives in innate immunity. Science 284: 1313-1318.

Irie, T., Muta, T., and Takeshige, K. 2000. TAK1 mediates an activation signal from toll-like receptor(s) to nuclear factor$\kappa \mathrm{B}$ in lipopolysaccharide-stimulated macrophages. FEBS Lett. 467: 160-164.

Ishitani, T., Ninomiya-Tsuji, J., Nagai, S., Nishita, M., Meneghini, M., Barker, N., Waterman, M., Bowerman, B., Clevers, H., Shibuya, H., et al. 1999. The TAK1-NLK-MAPKrelated pathway antagonizes signalling between $\beta$-catenin and transcription factor TCF. Nature 399: 798-802.

Khush, R.S. and Lemaitre, B. 2000. Genes that fight infection: What the Drosophila genome says about animal immunity. Trends Genet. 16: 442-449.

Khush, R.S., Leulier, F., and Lemaitre, B. 2001. Drosophila immunity: Two paths to NF-кB. Trends Immunol. 22: 260-264

Kim, Y.S., Han, S.J., Ryu, J.H., Choi, K.H., Hong, Y.S., Chung, Y.H., Perrot, S., Raibaud, A., Brey, P.T., et al. 2000. Lipopolysaccharide-activated kinase, an essential component for the induction of the antimicrobial peptide genes in Drosophila melanogaster cells. J. Biol. Chem. 275: 2071-2079.

Lemaitre, B., Kromer-Metzger, E., Michaut, L., Nicolas, E., Meister, M., Georgel, P., Reichhart, J., and Hoffmann, J. 1995. A recessive mutation, immune deficiency (imd), defines two distinct control pathways in the Drosophila host defense. Proc. Natl. Acad. Sci. 92: 9365-9469.

Lemaitre, B., Nicolas, E., Michaut, L., Reichhart, J., and Hoffmann, J. 1996. The dorsoventral regulatory gene cassette spätzle/Toll/cactus controls the potent antifungal response in Drosophila adults. Cell 86: 973-983.

Lemaitre, B., Reichhart, J., and Hoffmann, J. 1997. Drosophila host defense: Differential induction of antimicrobial peptide genes after infection by various classes of microorganisms. Proc. Nat1. Acad. Sci. 94: 14614-14619.

Leulier, F., Rodriguez, A., Khush, R.S., Chen, P., Abrams, J.M., and Lemaitre, B. 2000. The Drosophila caspase Dredd is required to resist Gram-negative bacterial infection. $E M B O$ Rep. 1: 353-358.

Levashina, E., Ohresser, S., Lemaitre, B., and Imler, J. 1998. Two distinct pathways can control expression of the Drosophila antimicrobial peptide Metchnikowin. J. Mol. Biol. 278: 515527.

Lu, Y., Wu, L.P., and Anderson, K.V. 2001. The antibacterial arm of the Drosophila innate immune response requires an ІкB kinase Genes \& Dev. 15: 104-110.

Manfruelli, P., Reichhart, J.M., Steward, R., Hoffmann, J.A., and Lemaitre, B. 1999. A mosaic analysis in Drosophila fat body cells of the control of antimicrobial peptide genes by the Rel proteins Dorsal and DIF. EMBO J. 18: 3380-3391. 
Meng, X., Khanuja, B.S., and Ip, Y.T. 1999. Toll receptor-mediated Drosophila immune response requires Dif, an NF-кB factor. Genes \& Dev. 13: 792-797.

Mihaly, J., Kockel, L., Gaengel, K., Weber, U., Bohmann, D., and Mlodzik, M. 2001. The role of the Drosophila TAK homologue dTAK during development. Mech. Dev. 102: 67-79.

Moriguchi, T., Kuroyanagi, N., Yamaguchi, K., Gotoh, Y., Irie, K., Kano, T., Shirakabe, K., Muro, Y., Shibuya, H., Matsumoto, et al. 1996. A novel kinase cascade mediated by mitogen-activated protein kinase kinase 6 and MKK3. J. Biol. Chem. 271: 13675-13679.

Ninomiya-Tsuji, J., Kishimoto, K., Hiyama, A., Inoue, J., Cao, Z., and Matsumoto, K. 1999. The kinase TAK1 can activate the NIK-I $\kappa \mathrm{B}$ as well as the MAP kinase cascade in the IL-1 signalling pathway. Nature 398: 252-256.

Onfelt Tingvall, T., Roos, E., and Engstrom, Y. 2001. The imd gene is required for local Cecropin expression in Drosophila barrier epithelia. EMBO Rep. 2: 239-243.

Rodriguez, A., Oliver, H., Zou, H., Chen, P., Wang, X., and Abrams, J.M. 1999. Dark is a Drosophila homologue of Apaf1/CED-4 and functions in an evolutionarily conserved death pathway. Nat. Cell Biol. 1: 272-279.

Rutschmann, S., Jung, A.C., Hetru, C., Reichhart, J.M., Hoffmann, J.A., and Ferrandon, D. 2000a. The Rel protein DIF mediates the antifungal but not the antibacterial host defense in Drosophila. Immunity 12: 569-580.

Rutschmann, S., Jung, A.C., Zhou, R., Silverman, N., Hoffmann, J.A., and Ferrandon, D. 2000b. Role of Drosophila IKK $\gamma$ in a Toll-independent antibacterial immune response. Nat. Immunol. 1: 342-347.

Sakurai, H., Miyoshi, H., Toriumi, W., and Sugita, T. 1999. Functional interactions of transforming growth factor $\beta$-activated kinase 1 with IкB kinases to stimulate NF- $\kappa$ B activation. J. Biol. Chem. 274: 10641-10648.

Sakurai, H., Shigemori, N., Hasegawa, K., and Sugita, T. 1998. TGF- $\beta$-activated kinase 1 stimulates NF- $\kappa$ B activation by an NF-к B-inducing kinase-independent mechanism. Biochem. Biophys. Res. Commun. 243: 545-549.

Shibuya, H., Iwata, H., Masuyama, N., Gotoh, Y., Yamaguchi, K., Irie, K., Matsumoto, K., Nishida, E., and Ueno, N. 1998. Role of TAK1 and TAB1 in BMP signaling in early Xenopus development. EMBO J. 17: 1019-1028.

Shirakabe, K., Yamaguchi, K., Shibuya, H., Irie, K., Matsuda, S., Moriguchi, T., Gotoh, Y., Matsumoto, K., and Nishida, E. 1997. TAK1 mediates the ceramide signaling to stress-activated protein kinase/c-Jun N-terminal kinase. J. Biol. Chem. 272: 8141-8144.

Silverman, N., Zhou, J., Stöven, S., Pandey, N., Hultmark, D., and Maniatis, T. 2000. A Drosophila IkB kinase complex required for Relish cleavage and antibacterial immunity. Genes \& Dev. 14: 2461-2471.

Stöven, S., Ando, I., Kadalayil, L., Engström, Y., and Hultmark, D. 2000. Activation of the Drosophila NF-кB factor Relish by rapide endoproteolytic cleavage. EMBO Rep. 1: 347-352.

Takatsu, Y., Nakamura, M., Stapleton, M., Danos, M.C., Matsumoto, K., O'Connor, M.B., Shibuya, H., and Ueno, N. 2000. TAK1 participates in c-Jun $\mathrm{N}$-terminal kinase signaling during Drosophila development. Mol. Cell. Biol. 20: 3015-3026.

Tzou, P., Ohresser, S., Ferrandon, D., Capovilla, M., Reichhart, J.M., Lemaitre, B., Hoffmann, J.A., and Imler, J.L. 2000. Tissue-specific inducible expression of antimicrobial peptide genes in Drosophila surface epithelia. Immunity 13: 737748.

Wang, W., Zhou, G., Hu, M.C., Yao, Z., and Tan, T.H. 1997. Activation of the hematopoietic progenitor kinase-1 (HPK1)- dependent, stress-activated c-Jun $\mathrm{N}$-terminal kinase (JNK) pathway by transforming growth factor- $\beta$ (TGF- $\beta$ )-activated kinase (TAK1), a kinase mediator of TGF- $\beta$ signal transduction. J. Biol. Chem. 272: 22771-22775.

Yamaguchi, K., Shirakabe, K., Shibuya, H., Irie, K., Oishi, I., Ueno, N., Taniguchi, T., Nishida, E., and Matsumoto, K. 1995. Identification of a member of the MAPKKK family as a potential mediator of TGF- $\beta$ signal transduction. Science 270: 2008-2011. 


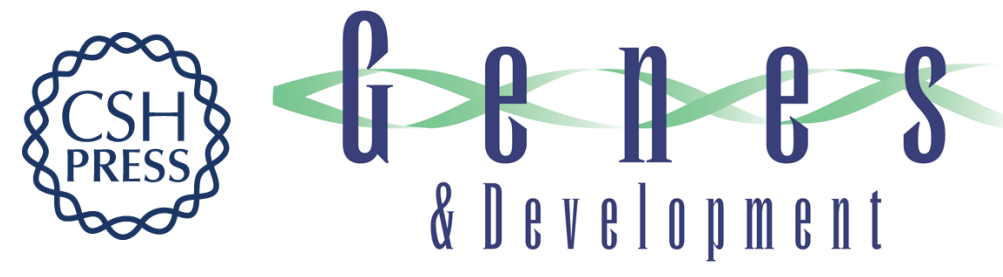

\section{Mutations in the Drosophila $d T A K 1$ gene reveal a conserved function for MAPKKKs in the control of rel/NF- $\kappa B$-dependent innate immune responses}

Sheila Vidal, Ranjiv S. Khush, François Leulier, et al.

Genes Dev. 2001, 15:

Access the most recent version at doi:10.1101/gad.203301

\section{References This article cites 45 articles, 20 of which can be accessed free at: http://genesdev.cshlp.org/content/15/15/1900.full.html\#ref-list-1}

License

Email Alerting Service right corner of the article or click here.

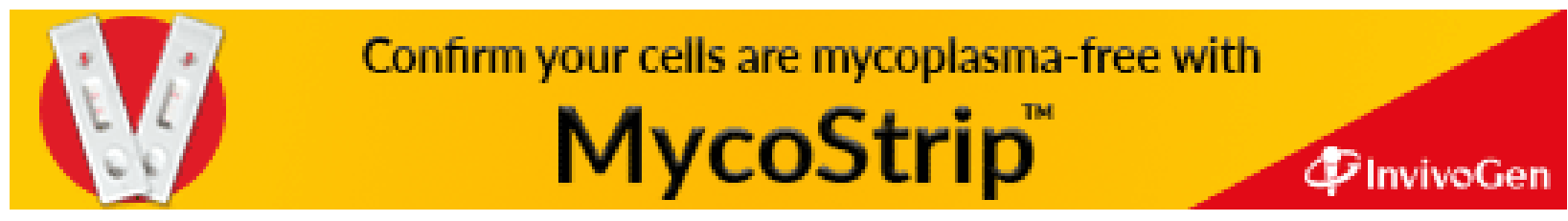

